\title{
Gridless ionized metal flux fraction measurement tool for use in ionized physical vapor deposition studies
}

\author{
T. G. Snodgrass, J. H. Booske, W. Wang, A. E. Wendt, and J. L. Shohet ${ }^{\text {a) }}$ \\ Engineering Research Center for Plasma-Aided Manufacturing, University of Wisconsin-Madison, \\ Madison, Wisconsin 53706
}

(Received 12 May 1998; accepted for publication 19 October 1998)

\begin{abstract}
Ionized physical vapor deposition is a technique for sputtering metal into small trenches, by ionizing sputtered metal atoms so that their trajectories can be controlled by electric fields. To this date no one has quantified exactly what fraction of the metal vapor is ionized, although the trends of how ionization varies with input parameters is known. This article describes and demonstrates a new quartz crystal microbalance design, which can be used to measure the ionized metal flux fraction arriving at the substrate location. Instead of using grids to repel ions as similar devices do, this analyzer works by applying a voltage bias to the front surface of the crystal in order to repel ions. A magnetic field adjacent to the face limits electron current to the microbalance, minimizing its perturbation of the plasma. The measurement tool described in this article does not suffer from complications caused by placing grids in front of the monitor and is an attractive method for characterizing ionized physical vapor deposition systems. Ion and neutral metal fluxes as a function of ionizer power are presented for an argon/copper discharge. (C) 1999 American Institute of Physics. [S0034-6748(99)03701-6]
\end{abstract}

\section{INTRODUCTION}

Magnetron sputtering, a physical vapor deposition process, has been widely used to deposit metal layers on semiconductor wafers. The deposition process, using magnetrons, results in a very broad angular velocity distribution of the depositing atoms and can result in very uniform planar films. However this process has difficulty in depositing films into deep structures. As the feature size on semiconductor wafers decreases, the aspect ratio (depth/width ratio of a feature) typically increases. Using conventional magnetron sputter deposition in this case, results in a closing off of the feature and the formation of a buried void.

Ionized sputtering addresses the filling or coating problem in the following manner: (1) the metal is sputtered from a sputtering target as in conventional magnetron sputtering. (2) The metal vapor passes through medium density plasma where it is partially ionized. (3) The partially ionized vapor deposits anisotropically on the substrate. The ions, being accelerated across the plasma sheath, are highly directional and deposit at nearly equal rates on the bottom of a deep feature and on the main surface of the wafer. The neutral atoms have an isotropic distribution and deposit more metal on the upper side walls of a feature and less on the bottom.

A metal vapor which is substantially ionized fills ${ }^{1-4}$ or coats ${ }^{5,6}$ high aspect-ratio features much better than does conventional physical vapor deposition. A measurement of the fraction of the deposition flux that is ionized is a critical step in the optimization of this process. The purpose of this work is to demonstrate a new design for a simplified quartz crystal microbalance that offers superior measurement capabilities

${ }^{\text {a)} E l e c t r o n i c ~ m a i l: s h o h e t @ e n g r . w i s c . e d u ~}$ of the ionized metal flux fraction, i.e., the fraction of the depositing metal flux that is ionized.

\section{A. Methods to measure ionized metal flux fraction}

Several researchers have measured the ionized metal flux fraction deposited by ionized physical vapor deposition primarily with the aid of a gridded-energy analyzer. Yamashita ${ }^{7}$ used a gridded energy analyzer in front of a substrate, biasing the grid to either repel or admit ions. By using profilometry to compare the deposition rate for samples with only neutral deposition to those with neutrals and ions, he was able to determine the ionized metal flux fraction. Rossnagel and Hopwood ${ }^{8,9}$ used a fully in situ method where they placed a gridded-energy analyzer in front of a quartz crystal microbalance. Similar to Yamashita's method, the grids can be biased to either admit or repel ions, but the deposition rate is measured in real time with the quartz crystal microbalance to determine the ionized metal flux fraction. Most recently Green et al. ${ }^{10}$ used a three-grid energy analyzer in front of a quartz crystal microbalance for similar measurements. In their apparatus, the quartz crystal microbalance was biased to the potential of the substrate.

\section{B. Disadvantages of gridded-energy analyzer}

There are several difficulties with gridded-energy analyzers, which can complicate the data analysis and limit when a gridded-energy analyzer can be used. A griddedenergy analyzer is not effective when the Debye length, $\lambda_{\mathrm{De}} \equiv\left(\epsilon_{0} k T_{e} / n e^{2}\right)^{1 / 2}$, the characteristic length for shielding of potentials in a plasma, is much less than the distance between grid wires. $\epsilon_{0}$ is the permittivity of free space, $k$ is Boltzmann's constant, $T_{e}$ the electron temperature, $n$ the plasma density, and $e$ the fundamental charge. The grids 
gradually coat and begin to close off over time. Some ions and neutrals are lost as they pass through the grids, and invariably this happens at different rates for ions and neutrals. The signal strength from the microbalance is reduced because the conductance through the grids is less than unity.

The maximum plasma density at which a gridded energy analyzer will work is limited to the density at which the plasma sheaths surrounding two adjacent wires no longer overlap, at which point plasma can penetrate the grid. This distance is approximately ten Debye lengths. In practical units

$$
L_{\text {overlap }}=10 \lambda_{\mathrm{De}}=10 \times 740 \sqrt{\frac{T_{e}}{n}} \mathrm{~cm},
$$

where $T_{e}$, the electron temperature, is in units of $\mathrm{eV}$ and $n$ the plasma density is in units of $\mathrm{cm}^{-3}$. For normal discharge conditions, $T_{e}$ is approximately $2.5 \mathrm{eV}$. This implies, for the $250 \mu \mathrm{m}$ grid spacing of Ref. 10, a maximum permissible plasma density of $2 \times 10^{11} \mathrm{~cm}^{-3}$. Typical plasma density for ionized physical vapor deposition can range from $10^{10}$ to $10^{13} \mathrm{~cm}^{-3}$.

Over time, the gridded-energy-analyzer grid wires will become coated and the transparency will change. If the grid spacing were $250 \mu \mathrm{m}$ and the grid-wire diameter were 94 $\mu \mathrm{m}$, one would expect the transparency to decrease $10 \%$, from $53 \%$ to $48 \%$, after about $13.5 \mu \mathrm{m}$ of deposition. Typical deposition thickness for filling contacts, vias, or trenches is $1 \mu \mathrm{m}$, which implies 13 trench fill operations can be done before a significant change in gridded-energy-analyzer calibration appears. Typical deposition thickness for doing conformal coatings on features (example for barrier layers) is hundreds of Angstroms, which implies that 1000 or so conformal coating depositions can be made before the grids begin to change. Typically, the maximum deposition on a single quartz crystal is $20 \mu \mathrm{m}$, which implies that the grids will clog before the crystal reaches maximum thickness. If the above analyzer were used in a system that deposits metal at a rate of 1000 Angstroms/min (a minimum rate for metal fill operations in the integrated circuit industry), the grids would show a significant transparency change after about two hours of operation. From the above numbers, it is shown that coating of the grids in a gridded energy analyzer by deposited metal should not influence the accuracy of measurements if they are changed regularly. Nevertheless, because of these requirements, coating of the grids is an inconvenience.

In the gridded region, the metal atoms and ions can collide with the background gas and/or the grids. These interactions affect the deposition rate of the ions and neutrals. It is likely however, that the effects will be different for ions and neutrals. For example, it is unclear whether ions or neutrals traverse the grids more easily, but there is likely to be a difference. If the grids were aligned so that all the holes were above one another, one would expect the ions to make it through all the grids with less loss, since their trajectories should be normal to the plane of the grids. Rossnagel et al. ${ }^{9}$ differentially pumped their gridded-energy-analyzer microbalance in order to reduce effects caused by metal ions or

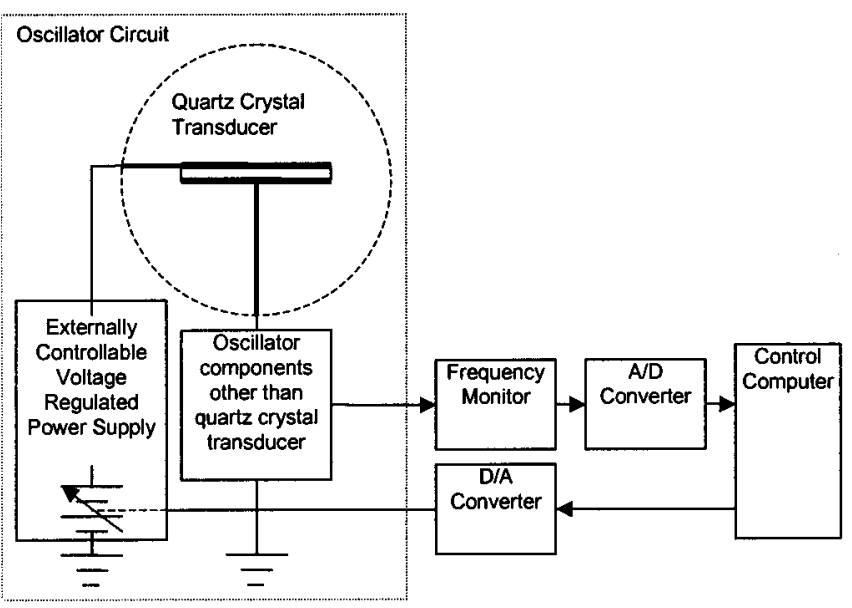

FIG. 1. Schematic diagram of quartz crystal microbalance circuit.

atoms colliding with the inert background gas, but it is unknown how this affected their measurements.

\section{INSTRUMENT DESIGN}

The measuring tool presented in this article eliminates grids entirely by biasing the front surface of the quartz crystal to repel ions. Typically the front surface (the surface exposed to deposition) of the crystal in a quartz crystal microbalance is coated with a thin gold or silver film upon which film is deposited. In a standard quartz crystal microbalance, the outer edge of the front surface then rests against the grounded surface of the crystal holder. The quartz crystal acts as a piezoelectric transducer in an oscillator circuit, in which the resonant frequency is dependent on the mass load on the quartz crystal (before any metal deposition the resonant frequency is typically $6 \mathrm{MHz}$ ). One side of the crystal is grounded, and the other side connected to the rest of the oscillator elements. In the instrument described here, the ground path from the crystal to the rest of the oscillator components is interrupted by a direct current (dc) voltage source (see Fig. 1). The dc voltage source has very little effect on the radio frequency (rf) signals in the oscillator circuit, and the oscillator operates as before with only a small change in frequency, which shifts the zero mass point slightly.

A magnetic field is used to protect the crystal from drawing a large electron saturation current when biased above the plasma potential to repel ions. If left unprotected, the high electron saturation current would heat the crystal and damage it. The magnetic field is oriented parallel to the surface of the crystal and localized just above the surface of the crystal. The magnetic field inhibits the diffusion of electrons to the substrate, because the electrons have very small cyclotron radii. However, the ions, which have much larger cyclotron radii, more easily cross the magnetic field lines. Not only should the magnetic field be chosen such that it has enough strength to inhibit the diffusion of electrons, but its region of effectiveness should be greater than the electron cyclotron radius and less than the ion cyclotron radius. In this instrument, the magnetic field is produced by a permanent magnet with its poles on either side of the crystal face. The magnetic 


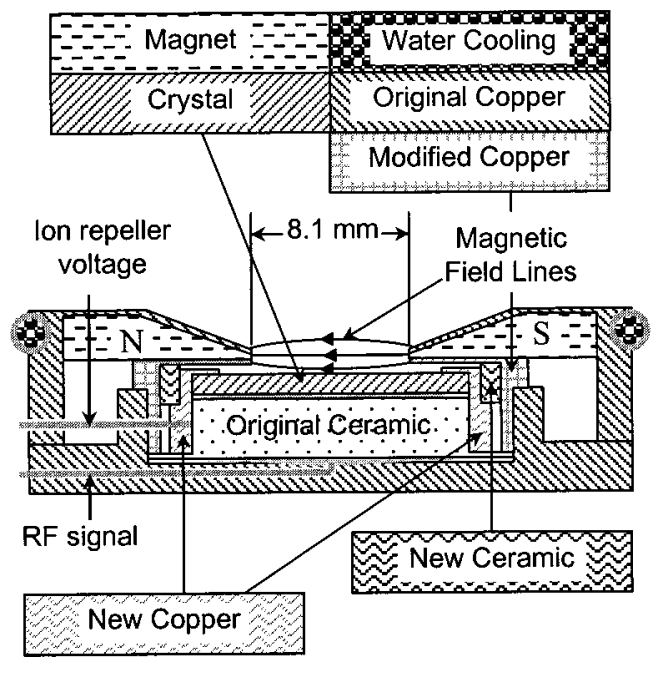

FIG. 2. Cross section of modified quartz crystal microbalance.

field is approximately $600 \mathrm{G}$ and peaks approximately $1 \mathrm{~mm}$ above the crystal face. For electrons with energy $E$ $=2.5 \mathrm{eV}$ (typical for an average electron in most plasma processing discharges) and with magnetic field $B_{0}=600 \mathrm{G}$, the orbital radius

$$
r_{c e}=\frac{3.37 \sqrt{E}}{B_{0}} \mathrm{~cm}=0.1 \mathrm{~mm} \text {. }
$$

Similarly $\mathrm{Cu}$ ions $(M=65 \mathrm{amu})$ given $E=10 \mathrm{eV}$ of energy by the plasma sheath have an orbital radius

$$
r_{c i}=\frac{144 \sqrt{E M}}{B_{0}} \mathrm{~cm}=6 \mathrm{~cm} .
$$

The specific instrument used to produce the results discussed in the next section was a modified Inficon XTC sputtering sensor. The sensor was modified such that a bias voltage can be applied to the front surface of the crystal as shown in Fig. 2. The original grounded electrode was hollowed out, and a ceramic spacer and a biasable electrode were placed inside the grounded part. The ceramic spacer is recessed as far as possible to prevent it from being coated with deposited film and shorting out the bias electrode. The sputter sensor originally came with an ALNICO 5 magnet used to protect the crystal from heating by the electrons from the plasma. Based on Eqs. (2) and (3) it was determined that this magnet could be used unaltered to protect the current device from the large electron currents as described above.

The frequency of the oscillator circuit is monitored by the Inficon XTC thin film thickness and deposition rate monitor, which produces an output voltage signal calibrated to be proportional to the deposition thickness on the crystal. The output from the thickness and deposition rate monitor and the input to the dc voltage supply are connected to a computer through a D/A A/D board. The computer was programmed to control the output voltage and read the deposition thickness from the monitor. It also calculates the deposition rate from the slope of the recorded deposition thickness versus time data.

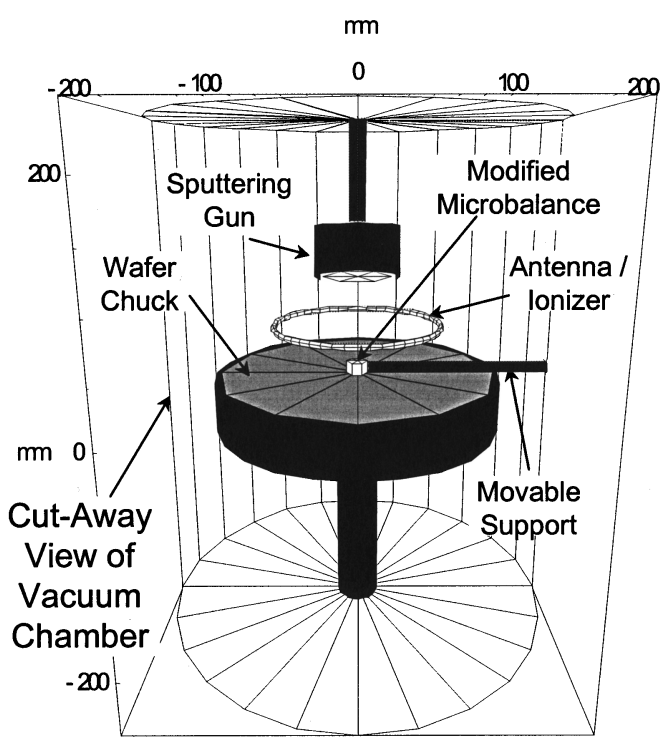

FIG. 3. Cut-away view of IPVD system vacuum chamber.

All experiments were done in a cylindrical ionized physical vapor deposition chamber with a single-turn copper antenna used as an ionizer (see Fig. 3). A Cu target provided the sputtered atoms. The instrument was placed on the axis of the cylinder and $100 \mathrm{~mm}$ below the target. Both water cooling and the sensor output signal are fed through a single 9.5-mm-o.d. stainless-steel tube. The tube slides in an O-ring seal to allow radial variation of the sensor location. The ionizer power supply can produce up to $600 \mathrm{~W}$ at $13.56 \mathrm{MHz}$. At $40 \mathrm{mTorr}$ of Ar and $600 \mathrm{~W}$ of radio frequency (rf) power to the ionizer, the plasma density is $5 \times 10^{11} \mathrm{~cm}^{-3}$ as measured with a Langmuir probe.

\section{RESULTS}

When the front crystal face is biased a few volts above the plasma potential, the ions no longer have enough energy to reach the crystal and only neutral atoms are deposited. Figure 4 shows the measured deposition rate as a function of the bias voltage to the front surface of the crystal. The plasma potential, measured with a Langmuir probe, was 9.4 $\mathrm{V}$. When the bias voltage was less than the plasma potential, all ions and neutrals are deposited making the deposition rate to the monitor independent of bias. Above the plasma potential, the deposition decreases as the ions are repelled, until a

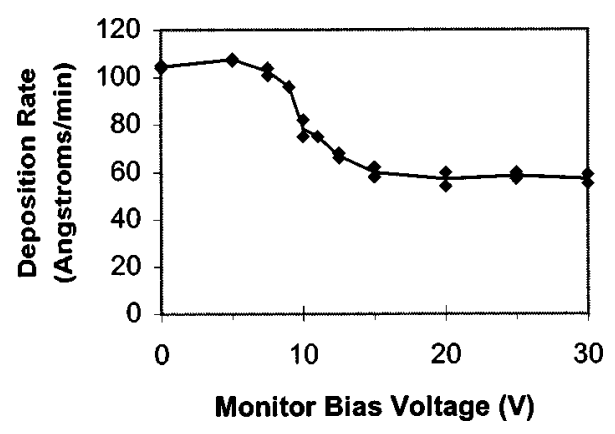

FIG. 4. Deposition rate as a function of crystal bias voltage. Operating conditions: 30 mTorr Ar, $400 \mathrm{~W}$ rf ionizer power, and $50 \mathrm{~W}$ dc sputter power of $\mathrm{Cu}$. 


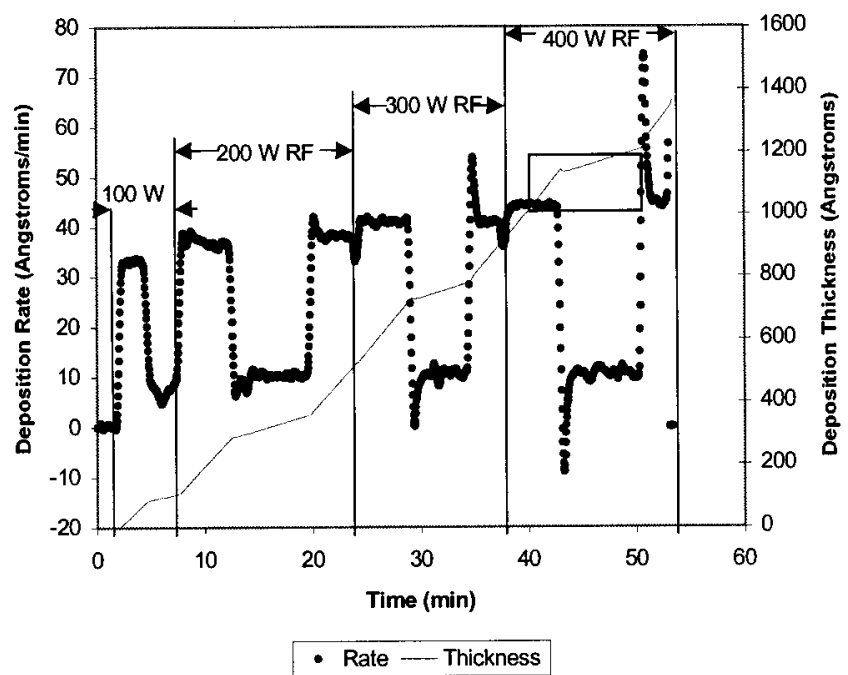

FIG. 5. Sample deposition rate vs time in 30 mTorr Ar plasma with $50 \mathrm{~W}$ dc sputtering of $\mathrm{Cu}$ at 100,200,300, and $400 \mathrm{~W}$ of rf power to the ionizer. At each power level, the instrument is biased at $0 \mathrm{~V}$ then to $30 \mathrm{~V}$ and back to $0 \mathrm{~V}$. The thin continuous line represents the raw detector signal, converted to deposition thickness vs time. The closed circles represent the deposition rate constructed by differentiating the thickness vs time curve.

bias of approximately $20 \mathrm{~V}$ has been reached at which point only neutral $\mathrm{Cu}$ atoms are being deposited. Beyond $20 \mathrm{~V}$ bias, the deposition rate is again constant, indicating that all of the ions have been repelled and only neutrals are being deposited. Thus, by measuring the deposition rate when ions and neutrals are deposited and subsequently measuring the deposition rate when only neutrals are being deposited, the ion deposition rate can be obtained by subtraction.

The ionized metal flux fraction can be measured as a function of background gas pressure, ionizer power (the rf power applied to the internal antenna), and sputter power. Figure 5 shows sample data of deposition rate versus time as measured by the instrument. After $2 \mathrm{~min}, 50 \mathrm{~W}$ of dc power is applied to the sputter source and held there for the duration of the measurement. The ionizer rf power is varied from 100 to $400 \mathrm{~W}$ in four steps as shown in the figure. At each ionizer power level, the bias on the crystal is changed from 0 to +30 $\mathrm{V} \mathrm{dc}$ and back to $0 \mathrm{~V}$ dc. Each time the crystal is biased to $+30 \mathrm{~V} \mathrm{dc}$ to repel ions, the deposition rate drops to approximately $10 \AA / \mathrm{min}$. When the crystal bias is reduced back to 0 $\mathrm{V} \mathrm{dc}$, the deposition rate returns to the rate before bias.

Every time the crystal is abruptly biased from 0 to $30 \mathrm{~V}$ or the ionizer power is increased, a dip in the instrument's output is recorded (see Fig. 6). This dip in the signal is a transient. It is probably due to the increased heat load put on the crystal and represents an offset in the "baseline" resonant frequency of the oscillator circuit. However the critical information is the deposition rate, rather than the instantaneous thickness of the accumulating metal film. Other than for the initial transient, this information, obtained as the derivative of the sensor's output signal, is unaffected by the differential-heat-load-induced dips. When the monitor bias is reduced from 30 to $0 \mathrm{~V}$, a transient increase in the deposition rate is observed. This is caused by a decrease in heat load on the crystal and is analogous to effect produced when the bias is increased causing an increase in heat load.

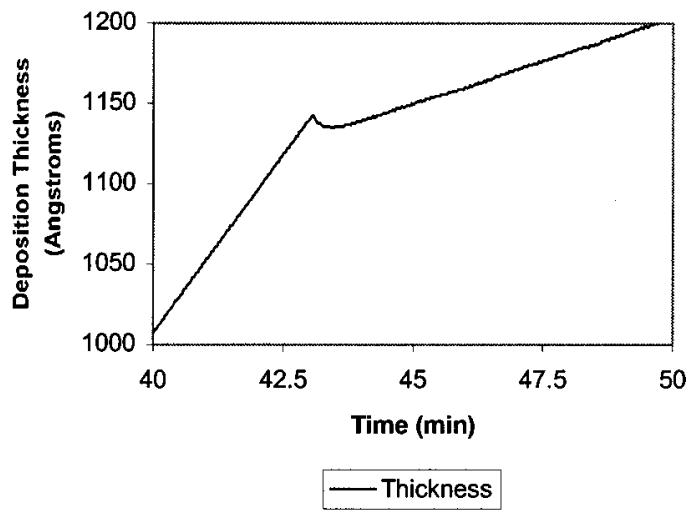

FIG. 6. Magnified region of Fig. 5, showing the dip in the detector output (at about $43 \mathrm{~min}$ ) associated with the increased heat load due to biasing the crystal face to $+30 \mathrm{~V}$.

\section{DISCUSSION}

Many measurements have been made with the instrument. In all measurements made, the precision in deposition rate could always be made to be within 1 Angstrom/min by ensuring sufficient measurement time (1-5 min). The instrument requires no calibration other than its original factory calibration. The sensitivity of the instrument is very high, because there are no grids to attenuate the deposition rate onto the crystal. The instrument employed in our system is very small, $35 \mathrm{~mm}$ diameter $\times 12 \mathrm{~mm}$ deep and mounted on a 9.5-mm-diam movable support shaft. The compact design also means that the instrument can be inserted quickly into any chamber with an ISO-KF40 port. Measurements have been made off axis in the system by moving the monitor support shaft radially through an O-ring feedthrough.

The instrument has a very low profile: $1.3 \mathrm{~mm}$ from the surface of the crystal to the outside of the housing. Comparing the $1.3 \mathrm{~mm}$ height to the $8.3 \mathrm{~mm}$ opening to the crystal gives an aspect ratio of 0.16 . This small aspect ratio implies that there is very little shadowing of the crystal by the surrounding structure and thus we can easily obtain a direct measurement of the depositing flux.

It should be pointed out that gridded analyzers, in order to accommodate multiple grids, require extra volume within the interior of the diagnostic. This leads to large-aspect-ratio (e.g., aspect ratio of 0.8 in Ref. 10) shadowing effects associated with different deposition patterns for the anisotropic ion and the isotropic neutral fluxes. Prior to converting the instrument's signals to proper flux fractions, one needs to perform additional calculations and calibration measurements to account for the different shadowing effects for ions and neutrals in instruments with gridded energy analyzers.

In contrast, the lack of grids on the device described in this article enables positioning of the microbalance deposition surface much closer to the entrance aperture surface, virtually eliminating shadowing and the need for concomitant calibration calculations or modeling to interpret the detector signals.

The limit of maximum density at which the instrument will work has not been reached to date. At some point, the 
portion of the electron current that leaks through the magnetic field will become too large and heat the crystal to the point where it will no longer function. The density limit of the present IPVD system has been $5 \times 10^{11} \mathrm{~cm}^{-3}$, which results in $6 \mathrm{~mA}$ of current to the crystal when biased at a +30 V.

An ionized metal flux fraction measurement diagnostic is described and demonstrated. The device uses a variable voltage bias supplied directly to the front surface of a quartz crystal microbalance to repel ions. This differs from other ionized metal flux fraction measurement tools in that it does not need any grids in front of the crystal to repel ions. The absence of grids makes for a more direct measurement of the ionized metal flux fraction than with a gridded tool and has been shown to work immersed in a plasma density as high as $5 \times 10^{11} \mathrm{~cm}^{-3}$.

\section{ACKNOWLEDGMENT}

This work supported by National Science Foundation Grant No. EEC-8721545.

${ }^{1}$ S. M. Rossnagel, J. Vac. Sci. Technol. B 13, 125 (1995).

${ }^{2}$ S. Hamaguchi and S. M. Rossnagel, J. Vac. Sci. Technol. B 13, 183 (1995).

${ }^{3}$ P. F. Chen, S. M. Rossnagel, and D. N. Ruzic, J. Vac. Sci. Technol. B 13, 203 (1995).

${ }^{4}$ S. M. Gorbatkin, D. B. Poker, R. L. Rhoades, C. Doughty, L. A. Berry, and S. M. Rossnagel, J. Vac. Sci. Technol. B 14, 1853 (1996).

${ }^{5}$ S. Hamaguchi and S. M. Rossnagel, J. Vac. Sci. Technol. B 14, 2603 (1996).

${ }^{6}$ C. A. Nichols, S. M. Rossnagel, and S. Hamaguchi, J. Vac. Sci. Technol. B 14, 3270 (1996).

${ }^{7}$ M. Yamashita, J. Vac. Sci. Technol. A 7, 151 (1989).

${ }^{8}$ S. M. Rossnagel and J. Hopwood, Appl. Phys. Lett. 63, 3285 (1993).

${ }^{9}$ S. M. Rossnagel and J. Hopwood, J. Vac. Sci. Technol. B 12, 449 (1994).

${ }^{10}$ K. M. Green, D. B. Hayden, D. R. Juliano, and D. N. Ruzic, Rev. Sci. Instrum. 68, 4555 (1997). 